\title{
Night blindness is a serious public health problem of pregnant women's in Tahtay Koraro District, Tigray region, Northern Ethiopia
}

\author{
Omer Seid ${ }^{1, *}$, Mache Tsadik ${ }^{1}$, Nechey Kassa ${ }^{2}$ \\ ${ }^{1}$ Department of Public Health, College of Health Science, Mekelle University, Mekelle, Ethiopia \\ ${ }^{2}$ College of Health Sciences, Axum University, Axum, Ethiopia \\ Email address: \\ seoumer@yahoo.com (O. Seid), machetsadik@yahoo.com (M. Tsadik), kassanechey@yahoo.com (N. Kassa)
}

\section{To cite this article:}

Omer Seid, Mache Tsadik, Nechey Kassa. Night Blindness is a Serious Public Health Problem of Pregnant Women's in Tahtay Koraro District, Tigray Region, Northern Ethiopia. Journal of Food and Nutrition Sciences. Vol. 3, No. 1, 2015, pp. 17-23.

doi: $10.11648 /$ j.jfns.20150301.13

\begin{abstract}
Introduction: Night blindness is significant public health problem among pregnant women in Ethiopia and estimated to be more severe in rural areas. However; there is insufficient information about the magnitude of night blindness among pregnant women's of rural Ethiopia. The same is true in the study region. Objective: To assess the prevalence and associated factors of night blindness among pregnant Women's in Tahtay Koraro District, Tigray region, Northern Ethiopia. Methods: Community based cross sectional study design was employed in February 2013 among selected 323 pregnant women's. Two stage cluster sampling was employed; in the first stage seven kebeles were selected randomly, in the second stage study subjects were selected with considering probability proportional to population size (PPS). A structured and pretested questionnaire was used for data collection based on the objective of the study. The data was entered, cleaned and analyzed using statistical package for social science (SPSS) version 16. Bivariate \& multivariate logistic regression analysis was done to identify factors associated with night blindness at confidence limits of $95 \%$ and P-value less than 5\% significant. Frequencies, proportion, summary statistics were used for presenting the result of the study. Results: out of 323 pregnant women $56(17 \%)$ were suffered from night blindness. Pregnant women's age beyond 35 years were 4.9 times more likely to be night blinded than those with age less than 25 years [AOR=4.9; 95\% CI: (1.6-14.9)]. Pregnant women's income less than 500 Ethiopian birr per month were 2 times more likely to be night blinded than those who can earn more than that $[\mathrm{AOR}=2.0 ; 95 \%$ CI:(1.02-3.9)]. Pregnant women's didn't have own cattle were 5.6 times more likely to be night blinded than those who have cattle $[\mathrm{AOR}=5.6 ; 95 \% \mathrm{CI}:(2.9,11.0)]$. Pregnant women's with no hand washing practice after toilet were 3 times more likely to be night blinded than having hand washing practice after toilet [AOR=3.0;95\% CI:(1.3 -7.1)]. Conclusion: Night blindness is a serious public health problem of pregnant women's. Pregnant Women's age greater than 35 years, not having cattle, no hand washing practice after toilet and income less than 500 Ethiopian birr per month, were significantly affected by night blindness.
\end{abstract}

Keywords: Pregnant Women's Night Blindness, Associated Factors, Tahtay Koraro District

\section{Introduction}

Maternal night blindness is the inability to see normally after dusk or at night during pregnancy, especially the last trimester and early postpartum. Night blindness is the most common ocular manifestation of moderate to severe Vitamin A deficiency (VAD). Rhodopsin, in the retinal receptors, is responsible for seeing under low levels of illumination (rods) and is evidenced by delayed dark adaptation or difficulty seeing in dim light $[1,2]$.
Maternal night blindness is an indicator of severe VAD. It is one of the two biological indicators of VAD recommended by WHO as determinants of public health problem. VAD occurs when vitamin A intake or liver stores fail to meet daily metabolic requirements and the most common cause is a persistently low intake of vitamin A rich foods; when there is a problem with absorption, conversion or utilization of vitamin $\mathrm{A}$; or when children suffer from repeated infections or diseases such as measles, diarrhea and acute respiratory infections. If a diet is lacking in oils or fats, vitamin A is not 
well absorbed and utilized [3].

Vitamin A is among the micronutrients needed during pregnancy and pregnant women are among the vulnerable groups for Night blindness when there is VAD. There are an estimated 19 million pregnant women affected with VAD globally and it has been estimated that more than 6 million women develop night blindness during pregnancy each year. The prevalence of night blindness is of moderate to severe public health significance in 66 countries for pregnant women. A comparable and high proportion of pregnant women affected by night blindness are in Africa (9.8\%) and South-East Asia (9.9\%), each of which is estimated to have over 3 million pregnant women affected [3, 4]. An estimated 10 percent of women in low-income regions of the world experience night blindness during pregnancy. In addition to the above a reported prevalence rate of night blindness among pregnant women of 5 percent or higher is considered a signal that VAD is a serious public health problem in a given population [2].

Ethiopia is among the countries with a significant public health night blindness problem among pregnant women who are at risk of VAD [5]. The national prevalence of night blindness among pregnant women conducted in 1999 was $22.1 \%$ [6]. As determined using history of night blindness during last pregnancy; in rural communities $(23.1 \%)$ of women reported previous night blindness, in Tigray region, the prevalence of previous night blindness among women of $15-49$ ages and pregnant is $24.9 \%$ and $31 \%$ respectively $[7,8]$.

Night blind women were more susceptible to illness, both infectious (e.g. symptoms of urinary and possibly reproductive tract infections, diarrhea and dysentery) and non-infectious (e.g. symptoms of pre-eclampsia or eclampsia, and poor appetite, nausea and vomiting $[9,10]$. All these are problems of Ethiopian pregnant women. On the other hand Night blindness is influenced by socioeconomic \& demographic factors, weekly food intake, weekly morbidity history \& other risk factors $[10,11,12]$. The determinant factor also estimated that night blindness is a public health problem of pregnant women in Ethiopia.

However, there is no enough evidence on the prevalence and risk factors of VAD among pregnant mothers of rural Ethiopia. The aim of the study was to assess the prevalence and associated factors of night blindness among pregnant Women's in Tahtay Koraro, Tigray region, Northern Ethiopia. So, that this study provides a clue about pregnant women's night blindness in rural Ethiopia and planners or programs may used as the result for their intervention or it provide a base line information for further studies accordingly.

\section{Methods}

\subsection{Study Area and Study Period}

Tahtay koraro is one of the administrative districts found in Tigray region, northern Ethiopia. The district has fourteen kebelles, four health centers and ten health posts. The estimated population of the district for the year 2012/13 was
79,805 (50.02\% female). Mothers of reproductive age and pregnant women in the district are estimated to be 18,666 and 2722 respectively according to Tahtay koraro District Health Office population progression Document, 2005 report. The study was conducted in February, 2013.

\subsection{Study Design}

Community based cross-sectional study design was employed.

\subsection{Study Population}

All pregnant women's who live permanently in the study area were the source population, whereas, sampled or selected pregnant women who live in the selected kebeles were the study population.

\subsection{Sample Size Determination}

By using single population proportion formula, assuming a $95 \%$ confidence interval, $5 \%$ margin of error and $31 \%$ proportion of previous night blindness among pregnant women in Tigray region is considered [8]. By considering $10 \%$ nonresponse rate the final sample size was 323 pregnant women.

\subsection{Sampling Procedure}

Two stage cluster sampling was used. First stage; seven kebeles were selected randomly from the total fourteen kebeles. Second stage; study participants were selected using simple random sampling procedure. Probability proportional to population size (PPS) was used to allocate the participants in the selected kebeles.

\subsection{Data Collection Procedure}

Data were collected using structured interview administered questionnaire based on the objective of the study. The questionnaire was first developed in English and then translated into local language "Tigrigna" and back to English to check its consistency. The overall data collection activity was coordinated by the principal investigator.

\subsection{Night Blindness}

Study subjects are considered as having night blindness when they answered "no" to question 1 and "yes" to question 2 for pregnant using WHO standard questions: (i) do you have difficulty seeing during the day?; (ii) do you have "hima" (a local term for night blindness)? [10,12]. Adequate Vit-A diet intake: when there is intake of vitamin A-rich foods such as animal origin foods and dark green leaves three or more times per week together. Poor consumption of Vit-A rich foods: in adequate intake of nutrients containing Vit-A rich foods per 24 hour and per week by respondents.

\subsection{Data Quality Management}

To ensure data quality a pre-tested structured questionnaire was used. Training was given for the data collectors and supervisors. On spot checking of the data collection 
procedure was made. The completeness of the questionnaire was checked every day and incomplete questionnaires were filed in the following day.

\subsection{Data Processing and Analysis}

The data was entered, cleaned and analyzed using SPSS version 16 statistical package. Frequency distribution, proportion, means \& standard deviation was employed to analyses unvaried characteristics. Bivariate \& multivariate logistic regression was done to identify the associated factors of pregnancy night blindness at confidence level of $95 \%$ and P-value of $5 \%$.

\subsection{Ethical Consideration}

Ethical clearance was obtained from the Institutional Review Board of College of Health Sciences, Mekelle University. The formal permission paper was obtained from Tigray Regional Health Bureau, Tahtay Koraro district health office and the study kebeles. The purpose and importance of the study was explained to the study participants. Verbal consent was obtained from participants before starting interviewing. Confidentiality of the information was maintained by not including their names and personal identification.

\section{Results}

\subsection{Socio-Demographic and Economic Characteristics of Pregnant Women's and Night Blindness}

In the study a total of 323 pregnant women (which is a response rate of $100 \%$ ) were participated. The mean age and age at first marriage of the respondents were $31.4 \pm 6.7$ and $17.9 \pm 2.7$ years respectively. Majority of the respondents (88.5\%) were Orthodox Christians in religion and the rest were Muslims. From the study participants $93.2 \%$ were married during the study time. Slightly above half of the respondents $(51.1 \%)$ were illiterate. Subsistence farming was the main occupation of pregnant mother and their husband. Seventeen percent of pregnant women were suffered from night blindness. Of these $50 \%, 41.1 \%$ and $9.8 \%$ were night blinded during $3^{\text {rd }}, 2^{\text {nd }}$ and $1^{\text {st }}$ trimester respectively. Majority of the study participants with night blindness (57.1\%) had not own cattle [Table-1].

Table 1. Socio-demographic and economic characteristics of pregnant women 's, Tahtay Koraro District, Tigray region, Northern Ethiopia, February 2013.

\begin{tabular}{|c|c|c|c|}
\hline \multirow{2}{*}{ Characteristics $(N=323)$} & & \multicolumn{2}{|c|}{ Night blindness } \\
\hline & & Yes $(\%)$ & No $(\%)$ \\
\hline \multirow{3}{*}{ Age in year } & $<25$ & $6(1.9)$ & $52(16.1)$ \\
\hline & $25-35$ & $26(8.0)$ & $131(40.6)$ \\
\hline & $>35$ & $24(7.4)$ & $84(26.0)$ \\
\hline \multirow{2}{*}{ Age at first marriage (in years) } & $<18$ & $23(7.1)$ & $113(35.0)$ \\
\hline & $\geq 18$ & $33(10.2)$ & $154(47.7)$ \\
\hline \multirow{2}{*}{ Religion } & Orthodox & $51(15.8)$ & $235(72.8)$ \\
\hline & Muslim & $5(1.6)$ & $32(9.9)$ \\
\hline \multirow{2}{*}{ Marital status } & Married & $48(14.9)$ & $253(78.3)$ \\
\hline & Others & $8(2.5)$ & $14(4.3)$ \\
\hline \multirow{2}{*}{ Maternal literacy } & Illiterate & $36(11.2)$ & $129(39.9)$ \\
\hline & Literate & $20(6.2)$ & $138(42.7)$ \\
\hline \multirow{2}{*}{ Husband literacy } & Illiterate & $27(8.4)$ & $93(28.8)$ \\
\hline & Literate & $29(9.0)$ & $174(53.9)$ \\
\hline \multirow{2}{*}{ Husband occupation } & Subsistence Farming & $52(16.1)$ & $229(70.9)$ \\
\hline & Others & $4(1.2)$ & $38(11.8)$ \\
\hline \multirow{2}{*}{ Mother occupation } & Subsistence Farming & $51(15.8)$ & $218(67.5)$ \\
\hline & Others & $5(1.6)$ & $49(15.2)$ \\
\hline \multirow{2}{*}{ Cattle ownership } & None & $32(9.9)$ & $71(22)$ \\
\hline & $\geq 1$ cattle & $24(7.4)$ & $196(60.7)$ \\
\hline \multirow{2}{*}{ Income in Ethiopian Birr } & $<500.00$ & $26(8.0)$ & $82(25.4)$ \\
\hline & $\geq 500.00$ & $30(9.3)$ & $185(57.3)$ \\
\hline \multirow{2}{*}{ Land ownership } & No & $12(3.7)$ & $31(9.6)$ \\
\hline & Yes & $44(13.6)$ & $236(73)$ \\
\hline \multirow{2}{*}{ Radio ownership } & Yes & $28(8.7)$ & $166(51.4)$ \\
\hline & No & $28(8.7)$ & $101(31.3)$ \\
\hline \multirow{3}{*}{ Distance to water source(min) } & Less than 15 & $21(6.5)$ & $90(27.9)$ \\
\hline & $15-30$ & $27(8.4)$ & $127(39.3)$ \\
\hline & $>30$ & $8(2.5)$ & $50(15.5)$ \\
\hline \multirow{3}{*}{ Hand wash } & Always & $9(2.8)$ & $85(26.3)$ \\
\hline & Sometimes & $15(4.6)$ & $60(18.6)$ \\
\hline & No & $32(9.9)$ & $122(37.8)$ \\
\hline \multirow{2}{*}{ Latrine availability } & Yes & $40(12.4)$ & $223(69.0)$ \\
\hline & No & $16(5.0)$ & $44(13.6)$ \\
\hline \multirow{2}{*}{ Waste disposal site } & Yes & $46(14.2)$ & $213(65.9)$ \\
\hline & No & $10(3.1)$ & $54(16.7)$ \\
\hline Prevalence of night blindness a & nant women's was $56(17 \%)$ & & \\
\hline
\end{tabular}




\subsection{Maternal and Children Characteristics}

Only, $12 \%$ of respondents have had greater than or equals two under five children. One out of two $(51.4 \%)$ of respondents were born three or more alive births. Above one forth $(22.3 \%)$ of pregnant women's were having history of greater than or equal to five total births. However, $60.7 \%$ of study participants have had three and above years birth spacing pattern. Only, $5.3 \%$ of study participates were having history of abortion [Table-2].

Table 2. Maternal and children characteristics of pregnant women's by night blindness status, Tahtay Koraro District, Tigray, Northern Ethiopia, February 2013

\begin{tabular}{|c|c|c|c|}
\hline \multirow{2}{*}{ Characteristics } & & \multicolumn{2}{|c|}{ Night blindness } \\
\hline & & Yes $(\%)$ & No $(\%)$ \\
\hline \multirow{2}{*}{$\begin{array}{l}\text { Number of under five } \\
\text { children }\end{array}$} & $<2$ & $50(15.5)$ & $234(72.4)$ \\
\hline & $\geq 2$ & $6(1.9)$ & $33(10.2)$ \\
\hline \multirow{2}{*}{ Birth spacing } & $<3$ years & $24(7.4)$ & $103(31.9)$ \\
\hline & $\geq 3$ years & $32(9.9)$ & $164(50.8)$ \\
\hline \multirow{2}{*}{$\begin{array}{l}\text { Number of children born } \\
\text { alive }\end{array}$} & $<3$ & $26(8.0)$ & $131(40.6)$ \\
\hline & $\geq 3$ & $30(9.3)$ & $136(42.1)$ \\
\hline \multirow{2}{*}{ Total number of births } & $<5$ children & $39(12.1)$ & $212(65.6)$ \\
\hline & $\geq 5$ & $17(5.3)$ & $55(17)$ \\
\hline \multirow{2}{*}{ History abortion } & Yes & $6(1.9)$ & $11(3.4)$ \\
\hline & No & $50(15.5)$ & $256(79.3)$ \\
\hline
\end{tabular}

\subsection{Food Frequency of Pregnant Women}

Seven day Food frequency of the pregnant women's was assessed. From the foods that were available, $79.9 \%, 23.2 \%$ and $52.3 \%$ of pregnant women's were consumed chilies, green leafy vegetable and fat fried foods three or more times per week respectively [Table-3].
Table 3. Food frequency of pregnant women's, Tahtay Koraro District, Tigray, Northern Ethiopia, February, 2013

\begin{tabular}{|c|c|c|c|}
\hline \multirow{2}{*}{ Frequency per week } & & \multicolumn{2}{|c|}{ Night blindness } \\
\hline & & Yes $(\%)$ & No $(\%)$ \\
\hline \multirow{2}{*}{ Maize } & $<3$ & $11(3.4)$ & $57(17.6)$ \\
\hline & $\geq 3$ & $45(13.9)$ & $210(65.0)$ \\
\hline \multirow{2}{*}{ Chilies } & $<3$ & $13(4.0)$ & $52(16.1)$ \\
\hline & $\geq 3$ & $43(13.3)$ & $215(66.6)$ \\
\hline \multirow{2}{*}{ Green leafy vegetables } & $<3$ & $41(12.7)$ & $207(64.1)$ \\
\hline & $\geq 3$ & $15(4.6)$ & $60(18.6)$ \\
\hline \multirow{2}{*}{ Porridge } & $<3$ & $33(10.2)$ & $148(45.8)$ \\
\hline & $\geq 3$ & $23(7.1)$ & $119(36.8)$ \\
\hline \multirow{2}{*}{ fat-fired foods } & $<3$ & $27(8.4)$ & $127(39.3)$ \\
\hline & $\geq 3$ & $29(9.0)$ & $140(43.3)$ \\
\hline \multirow{2}{*}{ Condiments } & $<3$ & $26(8.0)$ & $126(39.0)$ \\
\hline & $\geq 3$ & $30(9.3)$ & $141(43.7)$ \\
\hline
\end{tabular}

\subsection{Factors Associated with Night Blindness}

In the bivariate analysis; income less than 500 Birr, age greater than 35 years, functional latrine in-availability, marital status other than married, maternal illiteracy and ownership of cattle were found to be significantly associated with night blindness at $95 \%$ confidence level and $\mathrm{P}$ value $<0.05$ ). In the multivariate analysis, those with age beyond 35 years was 4.9 times more likely to be night blinded $[\mathrm{AOR}=4.9 ; 95 \% \mathrm{CI}$ : $(1.6-14.9)]$ than those with age less than or equal to 35 years. Women income less than 500 birr per month were 2.0 times more likely to be night blinded than those who can earn more than that $[\mathrm{AOR}=2.0$; 95\% CI:(1.02-3.9). Women who did not have cattle were 5.6 times more likely to have night blindness $[\mathrm{AOR}=5.6 ; 95 \% \mathrm{CI}$ : $(2.9,11.0)]$ than those who have cattle [Table-4].

Table 4. Factors associated with pregnant women's night blindness in Tahtay Koraro district, Tigray, northern Ethiopia, 2013.

\begin{tabular}{|c|c|c|c|c|c|}
\hline \multirow{2}{*}{ Characteristics } & & \multicolumn{2}{|c|}{ Night blindness } & \multirow{2}{*}{ COR (95\%CI) } & \multirow{2}{*}{ AOR (95\%CI) } \\
\hline & & Yes $(\%)$ & No $(\%)$ & & \\
\hline \multirow{2}{*}{ Age in year } & $<25$ & $6(1.9)$ & $52(16.1)$ & 1 & 1 \\
\hline & $>35$ & $24(7.4)$ & $84(26.0)$ & $2.5(0.9-6.5)$ & $4.9(1.6-14.9)$ \\
\hline \multirow{2}{*}{ Marital status } & Married & $48(14.9)$ & $253(78.3)$ & 1 & \\
\hline & Others & $8(2.4)$ & $14(4.3)$ & $3.0(1.2-7.6)$ & \\
\hline Maternal literacy & Literate & $20(6.2)$ & $138(42.7)$ & 1 & \\
\hline \multirow{2}{*}{ Husband literacy } & Illiterate & $27(8.4)$ & $93(28.8)$ & $1.7(1.0-3.1)$ & \\
\hline & Literate & $29(51.8)$ & $174(53.9)$ & 1 & \\
\hline \multirow{2}{*}{ Mother occupation } & Subsistence farming & $51(15.8)$ & $218(67.5)$ & $2.3(0.9-6.0)$ & \\
\hline & Others & $5(1.6)$ & $49(15.5)$ & 1 & \\
\hline Husband occupation & Subsistence farming & $52(16.1)$ & $229(70.9)$ & $2.2(0.7-6.3)$ & \\
\hline \multirow{2}{*}{ Cattle ownership } & None & $32(9.9)$ & $71(22.0)$ & $3.7(2.0-6.7)$ & $5.6(2.9-11.0)$ \\
\hline & $\geq 1$ & $24(7.4)$ & $196(60.7)$ & 1 & 1 \\
\hline \multirow{2}{*}{ Land ownership } & None & $12(3.7)$ & $31(9.6)$ & $2.1(1.0-4.4)$ & $2.3(1.0-5.5)$ \\
\hline & Own & $44(13.6)$ & $236(72.1)$ & 1 & 1 \\
\hline \multirow{2}{*}{ Radio ownership } & Yes & $28(8.7)$ & $166(51.4)$ & 1 & \\
\hline & No & $28(8.7)$ & $101(31.3)$ & $1.6(0.9-2.9)$ & \\
\hline \multirow{3}{*}{ Hand wash } & No & $32(9.9)$ & $122(37.8)$ & $2.5(1.1-5.5)$ & $3.0(1.3-7.1)$ \\
\hline & Sometimes & $15(4.6)$ & $60(18.6)$ & $2.4(1.0-5.8)$ & $2.3(0.9-6.2)$ \\
\hline & Always & $9(2.8)$ & $85(26.3)$ & 1 & 1 \\
\hline
\end{tabular}




\begin{tabular}{|c|c|c|c|c|c|}
\hline \multirow{2}{*}{ Characteristics } & & \multicolumn{2}{|c|}{ Night blindness } & \multirow{2}{*}{ COR $(95 \%$ CI $)$} & \multirow{2}{*}{ AOR $(95 \% C I)$} \\
\hline & & Yes $(\%)$ & No (\%) & & \\
\hline \multirow{2}{*}{ Latrine availability } & Yes & $40(12.4)$ & $223(69.0)$ & 1 & \\
\hline & No & $16(5.0)$ & $44(13.6)$ & $2.0(1.0-3.9)$ & \\
\hline \multirow{2}{*}{ Income in Ethiopian Birr } & $<500.00$ & $26(8.0)$ & $82(25.4)$ & $2.0(1.1-3.5)$ & $2.0(1.02-3.9)$ \\
\hline & $\geq 500.00$ & $30(9.3)$ & $185(57.3)$ & 1 & 1 \\
\hline \multirow{2}{*}{ Total number of births } & $<5$ children & $39(12.1)$ & $212(65.6)$ & 1 & \\
\hline & $\geq 5$ children & $17(5.3)$ & $55(17.0)$ & $0.6(0.3-1.1)$ & \\
\hline \multirow{2}{*}{ History of abortion } & Yes & $6(1.9)$ & $11(3.4)$ & $2.8(1.0-7.9)$ & \\
\hline & No & $50(15.5)$ & $256(79.3)$ & 1 & \\
\hline
\end{tabular}

\section{Discussion}

Maternal night blindness is common during pregnancy in many developing countries. Globally, night blindness affects 9.8 million women which correspond to $7.8 \%$ of the population at risk of VAD. A comparable and high proportion of women affected by night blindness are in Africa (9.8\%) and South-East Asia (9.9\%), each of which is estimated to have over 3 million pregnant women affected, or one third of the women affected globally [3, 4,5]. In this study, $17.3 \%$ of pregnant women's were suffered from night blindness and majority of them night blinded during the $3^{\text {rd }}$ and $2^{\text {nd }}$ trimester. This prevalence is above three times the cut of point of public health importance of WHO definition of night blindness (night blindness cut of point $\geq 5 \%$ ) [10] and above two times the global prevalence $(7.8 \%)$ and slightly below two times prevalence in Africa (9.8\%) and South-East Asia (9.9\%) however, it is below the national prevalence of 1999 and the national rural prevalence and regional prevalence $(22.1 \%),(23.1 \%)$ and $(24.9 \%)$ respectively [3, 4, $6,8]$. WHO report that, Ethiopia is among the developing countries by having significant night blinded pregnant women [5]. The finding in Tahtay Koraro approved this WHO report.

Different new evidences approved that VAD increases the risk of maternal death. Ethiopia is one of the tope courtiers by maternal mortality; the maternal mortality ratio (MMR) in country has stagnated at 676 per 100,000 live births in 2011 and 673 in 2005, even slight increment between 2005 to $2011[13,14]$. In this case, VAD could be a fundamental cause for maternal mortality. This is because night blind pregnant women are more susceptible to illness, both infectious (e.g. symptoms of urinary and possibly reproductive tract infections, diarrhea and dysentery) and non-infectious (e.g. symptoms of pre-eclampsia or eclampsia, and poor appetite, nausea and vomiting $[9,15,16]$. All this VAD related problems supported with poor accessibility and utilization of health services yields high prevalent of maternal mortality in Ethiopia.

VAD occurs when vitamin A intake or liver stores fail to meet daily metabolic requirements and the most common cause is a persistently low intake of vitamin A-rich foods; when there is a problem with absorption, conversion or utilization of vitamin $\mathrm{A}$; or when children suffer from repeated infections or diseases such as measles, diarrhea and acute respiratory infections (ARI). If a diet is lacking in oils or fats, vitamin A is not well absorbed and utilized. Others like Scio-demographic and economic factors also contribute a lot for vitamin A deficiencies and its clinical form night blindness.

In the current study of multivariate analysis, pregnant woman greater than 35 year old were 4.5 times night blinded than below 25 years old of their counterpart and the risk of developing night blindness increase with age (Table-4). Likely, a study from rural Terai of Nepal also suggested that women with age over 30 years were highest risk for night blindness than those women who are below this age range [17]. This may be due to frequent pregnancies and giving birth depletes the body vitamin A store or due to frequent pregnancies, there could be iron deficiency aneamia which affects hepatic retinol metabolism.

Regarding socioeconomic status, pregnant woman having income of less than 500 birr per month were 2 times night blinded than having income of equal or greater than 500 Ethiopian birr per month. It is consistent with a study in rural Cambodia which reports pregnant mothers with lower socioeconomic status are at higher risk for night blindness than those with higher socio economic-status [18]. This could be due to those who have high socio-economic states can get or consume vitamin A rich foods than the poorest and/or may have economically strong woman's may have used health services than the poor.

In the meantime woman's having cattle and farm land were 5.6 and 2.3 times less likely to be night blinded than woman's not having cattle and farm land respectively. It could be due to having cattle helps the mother to get animal source of foods easily and being having farm land relates to grading and consumption of vitamin A rich vegetables and fruits. Home gardening has been the most popular food-based strategy for the control of vitamin A deficiency, and various reviews have been published over the past 10 to 20 years [19] In Ethiopia, a home gardening and health and nutrition education intervention was built on to a previous dairy goat project. The participants increased their knowledge, attitude and practices related to vitamin A, child feeding practices and the prevention of night blindness [20]. In general nutrition sensitive strategy such as livestock production and home grading of vegetables and fruits are the well known sustainable interventions to tackle malnutrition and improves the socio-economic states of the households.

In other words nutrition sensitive intervention also improves the dietary diversity score of the mother's. DDS 
aim to reflect nutrient adequacy [21, 22, 23]. Studies in different age groups have shown that an increase in individual dietary diversity score is related to increased nutrient adequacy of the diet and this might suggest that there was no difference on the accessibility of the food intake at house hold level. FAO stated that, dietary diversity is a qualitative measure of food consumption that reflects household access to a variety of foods, and is also a proxy for nutrient adequacy of the diet of individuals [24].

Those woman's washed their hand after toilet were 3 times less likely night blinded than their counter part not washed after toilet. The conceptual frame work of UNICEF shows that, poor sanitation and hygiene is the well defined under line cause for malnutrition. Lack and not using toilet can influence malnutrition in two ways: first, households with no toilet facility are highly prone to infections due to contaminations that subsequently lead to disease-induced malnutrition. Secondly, a lack of toilet facility in the household can serve as a proxy to poor economic status, limited access to food, which in turns linked to higher malnutrition.

\section{Conclusion and Recommendation}

Night blindness is the most important public health problem of pregnant woman's. Pregnant women's of age greater than 35 years, not having cattle ownership, no hand washing practice after toilet and income less than 500 birr per month were significantly affected by night blindness. So, improving women's economic states is an important intervention to tackle maternal night blindness.

\section{References}

[1] Sand ford-Smith. Journal of Eye Diseases in Hot Climate 4th ed. London: Elsevier; 2003.

[2] International Vitamin Consultative Group. Maternal night blindness: a new indicator of vitamin A deficiency. IVACG statement 2002.

[3] Global Database on Vitamin A Deficiency. [database on the Internet]. DHS Statcompiler.2007 [cited 2/12/2012]. Available from: http://www.statcompiler.com/index.cfm.

[4] World Health Organization. Global Database on Vitamin A Deficiency. WHO 2009.

[5] World Health Organization. Global prevalence of vitamin A deficiency. In: System MDI, editor. Geneva,Switzerland 2007.

[6] Haider Jemal, Demissie Tesssma. Malnutrition and xerophthalmia in rural communities of Ethiopia. East African Medical jornal1999; 76:590-3.

[7] Haider Jemal, Demisse Tessma, G/Sillasie H, Fufa H, I Biratu. Vitamin A deficiency status in Tigray region, Ethiopia. Ethiopian Journal of Health Devevelopment 1999; 13:87-91.

[8] World Health Organization. data base on prevalence of previous night blindness in Tigray region conducted in 2000
[9] Christian P, West KJ, Khatry S, Pradhan E, LeClerq S, Katz J, et al. Night blindness during pregnancy and subsequent mortality among women in Nepal: effects of vitamin A and beta carotene supplementation. Am J Epidemiology2000; 152:542-7.

[10] World Health Organization. Indicators for Assessing Vitamin A Deficiency and Their Application in Monitoring and Evaluating Intervention Programmes. Micronutrient: WHO; 1996.

[11] World Health Organization. Guideline: vitamin A. supplementation in pregnant women. WHO2011.

[12] Christian P. Recommendations for Indicators: Night Blindness during Pregnancy; A Simple Tool to Assess Vitamin A Deficiency in a Population. Journal Nutrition 2002; 132:28848.

[13] Central Statistical Authority [CSA] and ORC Macro, Ethiopia Demographic and Health Survey 2005. Addis Ababa and Calverton, Maryland: CSA and ORC Macro. 2006.

[14] Central Statistical Authority [CSA] and ORC Macro, Ethiopia Demographic and Health Survey. Ethiopia Addis Ababa2011.

[15] Sommer A, Davidson F. Assessment and control of vitamin A deficiency: the Annecy accords. Journal of Nutrition. 2002; 132: $2845-50.27$

[16] Keith P, West J. Extent of Vitamin A Deficiency among Preschool Children and Women of Reproductive Age. J Nutr. 2002; 132: 2857-66.

[17] Katz J, Khatry S, West KJ, Humphrey J, LeClerq S, Pradhan E, et al. Night blindness is prevalent during pregnancy and lactation in rural Terai Nepal. Jornal Nutrition. 1995; 125: 2122-7.

[18] Semba R,de Pee S, Panagides D, Poly O, Bloem M. Risk factors for night blindness among women of childbearing age in Cambodia. European Journal of Clinical Nutrition2003; 57:1627-32.

[19] Peduzzi, C., Home and community gardens assessment program implementation experience: The tip of the iceberg. Vitamin A Field Support Project (VITAL) Report No. TA-2, ISTI, Washington, DC, 1990.

[20] Ayalew, W. Z., Wolde Gebriel, and Kassa, H., Reducing vitamin A deficiency in Ethiopia: Linkages with a womenfocused dairy goat farming project, OMNI Research Report Series No. 4, International Center for Research on Women, Washington, DC, 1999.

[21] Mirmiran, P., Azadbakht, L., Esmaillzadeh, A. \& Azizi, F. 2004. Dietary diversity score in adolescents- a good indicator of the nutritional adequacy of diets: Tehran lipid and glucose study. Asia Pacific Journal of Clinical Nutrition 13(1): 56-60.

[22] Foote, J., Murphy, S., Wilkens, L., Basiotis, P. \& Carlson, A. 2004. Dietary variety increases the probability of nutrient adequacy among adults. Journal of Nutrition 134: 1779-1785.

[23] Arimond, M., Torheim, L.E., Wiesmann, D., Joseph, M. \& Carriquiry A. 2009. Dietary Diversity as a Measure of the Micronutrient Adequacy of Women's Diets: Results from Rural Bangladesh Site. Washington (DC): Food and Nutrition Technical Assistance II Project, Academy for Educational Development;2009.(availableathttp://www.fantaproject.org/do wnloads/pdfs/WDDP_Bangladesh_Dec09.pdf) 
[24] Gina Kennedy, Terri Ballard and MarieClaude Dop. 2013. Guidelines for Measuring Household and Individual Dietary Diversity. Nutrition and Consumer Protection Division, Food and Agriculture Organization of the United Nations, Rome, Italy.2010. 